\title{
Endodontic treatment of tooth with morphological anomalies using cone-beam computed tomography
}

\author{
Sung-Hyeon Choi ${ }^{1 \dagger}$, Na-Kyung Yoon ${ }^{1 \dagger}$, Ji-Hyun Jang ${ }^{2}$, Young-Hoon Kim${ }^{1}$, Hoon-Sang Chang ${ }^{1}$, Yun-Chan \\ Hwang ${ }^{1}$, In-Nam Hwang ${ }^{1}$, Won-Mann $\mathrm{Oh}^{1}$, and Bin-Na Lee ${ }^{1 *}$ \\ ${ }^{1}$ Department of Conservative Dentistry, School of Dentistry, Dental Science Research Institute, Chonnam National Uni- \\ versity, Gwangju 61186, Republic of Korea \\ ${ }^{2}$ Department of Conservative Dentistry, School of Dentistry, Kyung Hee University, Seoul 02447, Republic of Korea
}

(Received Jan 29, 2018; Revised version received Jan 31, 2018; Accepted Feb 12, 2018)

\begin{abstract}
The aim of this study is to describe the endodontic treatment of a radix entomolaris (RE) with the C-shaped canal in mandibular first molar and a fused maxillary premolar with two supernumerary teeth with the separated root canal using cone-beam computed tomography imaging (CBCT). In our cases, non-surgical endodontic treatment based on the results of CBCT diagnosis, ProTaper rotary system, hand instrumentation, and passive ultrasonic irrigation along with periodontal treatment resulted in long-term and successful prognosis. Therefore, CBCT analysis an effective diagnostic tool for the treatment of teeth containing complex root canals.
\end{abstract}

KEY WORDS: Cone-beam computed tomography (CBCT), C-shaped canal, Fusion, Radix entomolaris, Supernumerary tooth

\section{Introduction}

Successful root canal treatment (RCT) depends on negotiation, irrigation, shaping and three-dimensional filling of canals. Traditionally, endodontic failures are associated with extra roots or missed canals [1]. Endodontic failure is linked to the presence of untreated canals [2]. Therefore, variations in root canal system play a key role in successful RCT. The most common variation in the mandibular molar is the presence of a distolingual supernumerary root called radix entomolaris (RE) in the mandibular first molar [3], or C-shaped canals in the mandibular second molar. Separate RE in the mandibular first molar has been detected in certain ethnic groups. A maximum frequency of $3 \%$ is found in African populations, while the frequency is less than $5 \%$ in Eurasian and Indian populations [4,5]. RE occurs with a frequency

\footnotetext{
These authors contributed equally to this work.

*Corresponding author: Bin-Na Lee

Department of Conservative Dentistry, School of Dentistry, Chonnam National University, 77 Young-bong ro, Buk-gu, Gwangju 61186, Republic of Korea

Tel.: +82-62-530-5868, Fax: +82-62-530-5629

E-mail: bnlee13@jnu.ac.kr
}

ranging from $5 \%$ to more than $30 \%$ in Mongoloid population [6-8]. The prevalence of C-shaped canals in Koreans has been reported to be as high as $32.7 \%$ and this variation is most frequently found in the mandibular second molar [9]. Although studies have reported RE or C-shaped root canals in mandibular molars, few studies have investigated the Cshaped root canal in mandibular first molar with RE [3-10].

Gemination and fusion are similar developmental anomalies of hard tissues with a different etiology [9]. Fusion is the union of hard tissue between two developing teeth. Fused teeth may share a common pulp chamber or have separate root canals and chambers. Gemination occurs due to an unsuccessful division of tooth germ, which usually leads to a single root, root canal, and a large bifid crown with a common pulp chamber [11]. It is more common in the anterior region, with approximately $0.1 \%$ occurring in permanent and $0.5 \%$ in primary dentition, with equal distribution among Caucasian males and females [12]. Most reported cases of fusion involve incisors and only rarely relate to posterior dentition [13-17].

Although conventional radiographic examination provides essential information for the successful management of endodontic problems, the technique has limited value due to the 
superimposition of anatomical structures [17]. Cone-beam computed tomography (CBCT) offers three-dimensional images of tooth at a relatively higher resolution and a lower dose. CBCT is therefore, the imaging modality of choice in challenging situations demanding localization and characterization of root canals [18]. Therefore, CBCT is an important tool for the evaluation of the root canal systems for successful endodontic treatment, especially in the case of teeth with complex root canal morphology [19-21].

Our case reports illustrate endodontic treatment of RE with a C-shaped canal in the mandibular first molar and a fused maxillary premolar with two supernumerary teeth containing separated root canal following CBCT imaging.

\section{Case Reports}

\section{Case 1}

A 46-year-old male patient was referred from a local
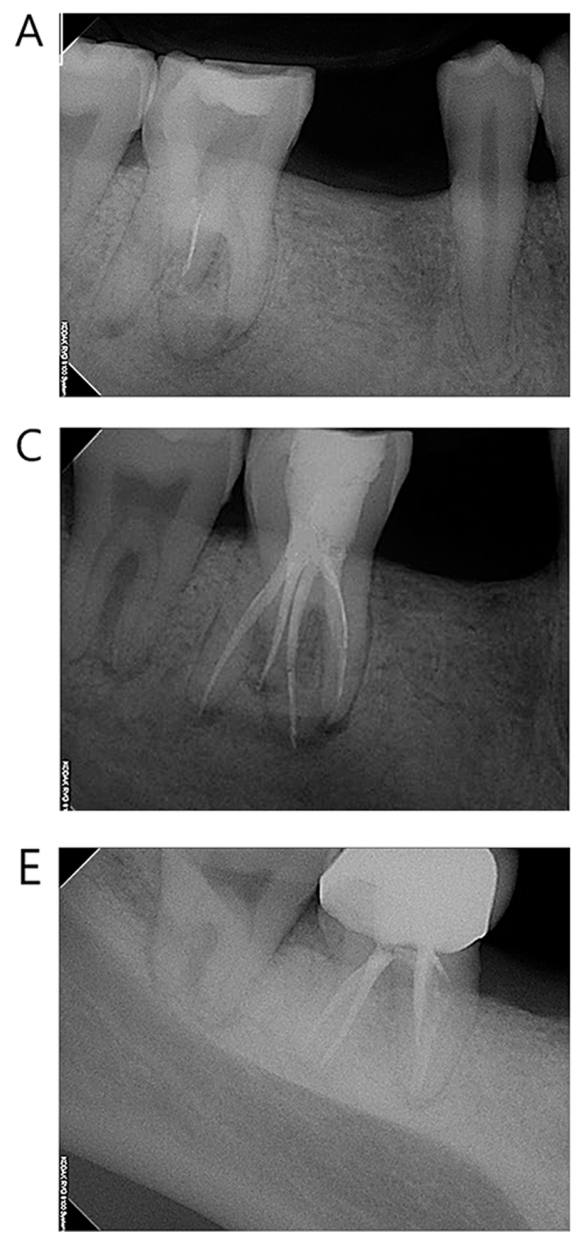

dental clinic to the Department of Conservative Dentistry at Chonnam National University Dental Hospital for evaluation and treatment of the right mandibular first molar (tooth \#46). The patient's chief complaint involved pain associated with the right mandibular first molar. The patient had a history of mental retardation. Clinical examination showed swelling of \#46 buccal gingival area and immobility of \#46. Cold test and electric pulp test elicited negative reaction. The tooth \#46 was sensitive to percussion. The mesiobuccal probing depth on \#46 was $10 \mathrm{~mm}$. Pus discharge via gingival sulcus of the \#46 mesiobuccal root was observed. Periapical radiograph suggested periapical radiolucency and endodontic file separation in the distolingual canal (Fig. 1A). To identify the root canal system thoroughly for prompt intervention, we performed a CBCT imaging of the tooth \#46 after obtaining informed consent from the patient and his parents (Alphard VEGA, Asahi Roentgen Ind. Co., Kyoto, Japan). A tube voltage $80 \mathrm{kV}$ and a tube current of $8 \mathrm{~mA}$ were used for CBCT.

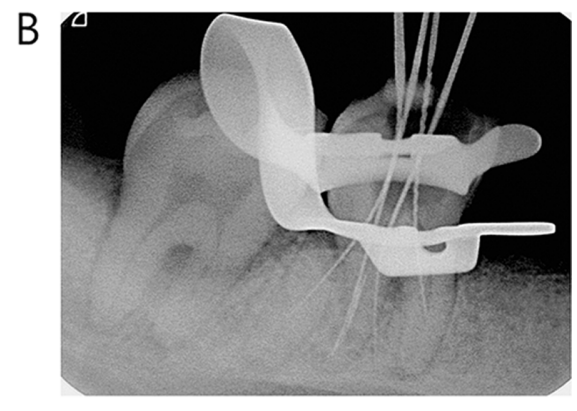

D

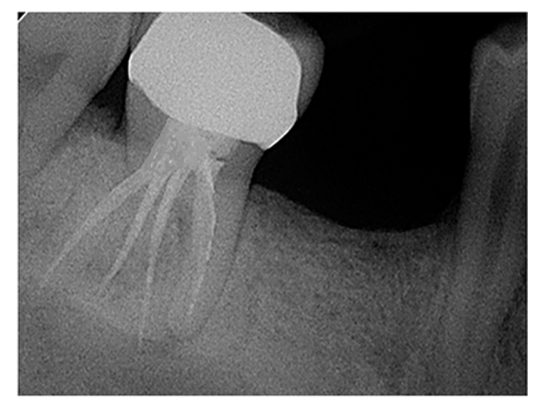

Fig. 1. (A) Preoperative periapical radiograph of the mandibular right first molar (\#46); (B) working length determination of \#46. (C) Postoperative periapical radiograph; (D) 9-month follow-up radiograph; (E) 18-month follow-up radiograph. 


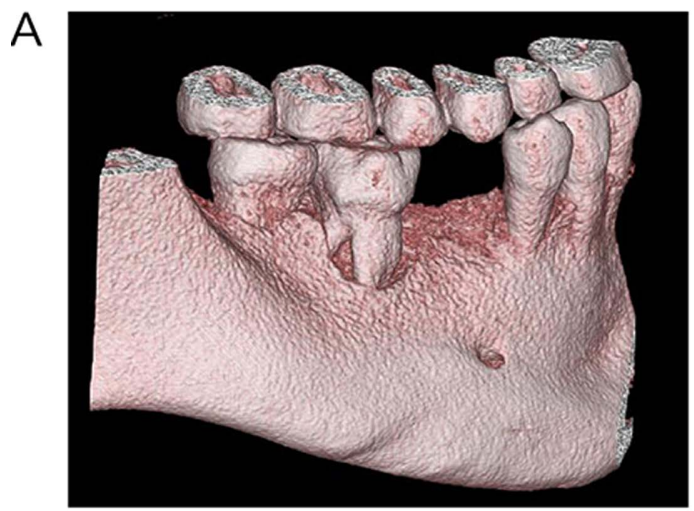

B
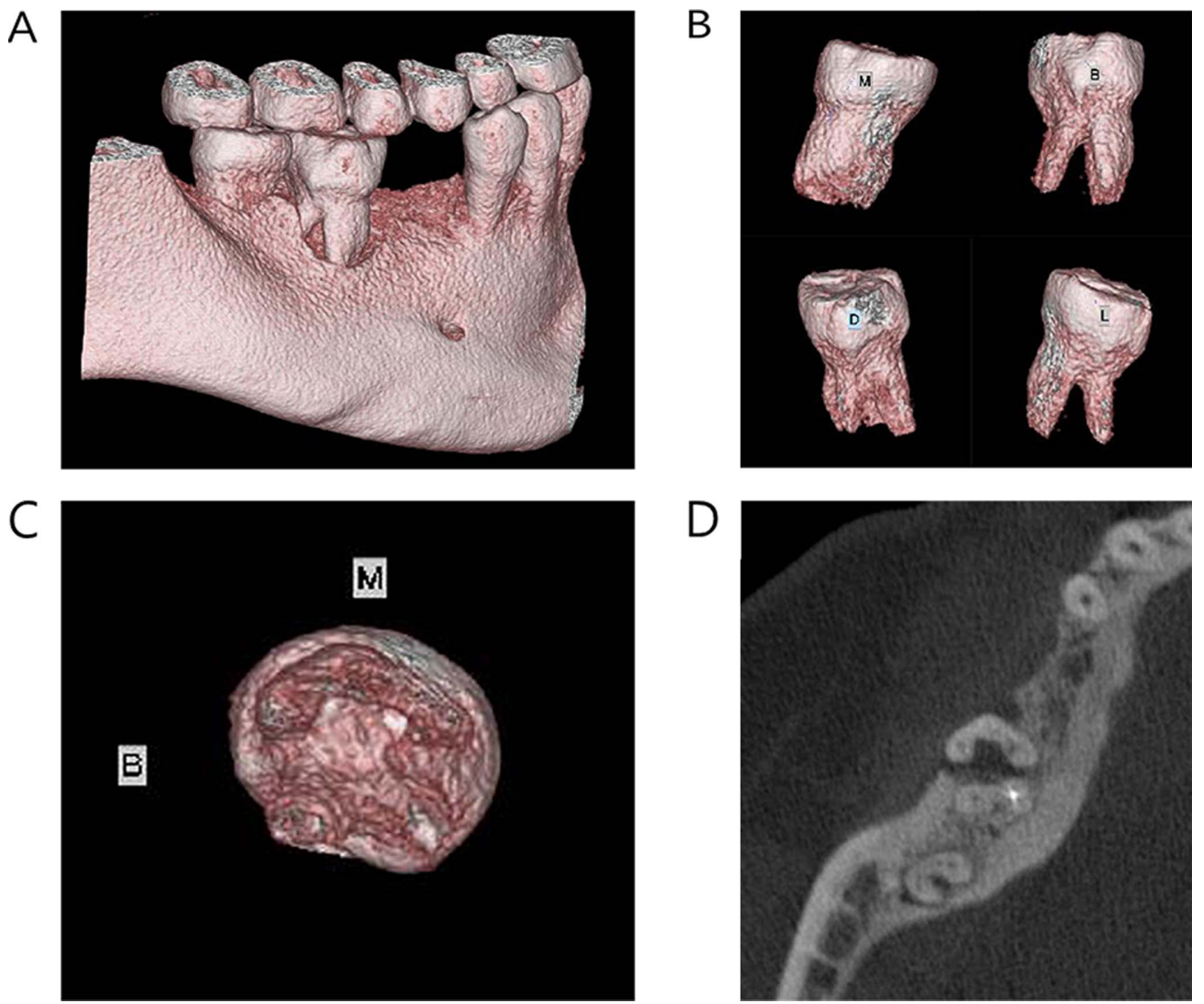

Fig. 2. CBCT images of \#46 confirming the presence of a separated RE and C-shaped canal. (A-C) 3-dimensional reconstruction images. (D) Axial image of the apical third presenting a C-shaped canal configuration.

The CBCT scan slices confirmed the presence of RE, the $\mathrm{C}$-shaped buccal canal and two apical foramina at the apical third (Fig. 2D). Based on these findings, the \#46 was diagnosed with periapical abscess with sinus to oral cavity. We decided to perform conventional root canal treatment with periodontal root planing.

The endodontic access was prepared under rubber dam isolation. The conventional triangular outline of the access cavity was modified into a trapezoidal form to position the distolingually located RE. The working length was estimated using electronic apex locator (Denta Port Root ZX, J Morita USA, Irvine, CA, USA) and confirmed using a periapical radiograph. We estimated the working length in the distolingual canal after bypassing the separated endodontic file in distolingual canal (Fig. 1B). All the canals were cleaned and shaped using ProTaper NiTi rotary instruments (Protaper universal, dentsply Dentsply-Maillefer, Ballaigues, Switzerland) with a crown-down technique and endodontic K-files (M access, Dentsply-Maillefer). Copious irrigation was performed using a $5.25 \%$ sodium hypochlorite $(\mathrm{NaOCl})$ solution and sterile saline using ultrasonic irrigation tip (Irrisafe, Acteon Satelec, GmbH, Germany) according to the manufacturer's instructions. The canals were dried with paper points and the access cavity was temporarily sealed with Caviton (GC Corporation, Tokyo, Japan). Periodontal root planing was performed on \#46.

After 1 week, the pain disappeared and gingival swelling subsided. We performed copious irrigation using $5.25 \% \mathrm{NaOCl}$ solution and sterile saline using an ultrasonic irrigation tip (Irrisafe, Acteon Satelec). The canals were dried with paper points and filled with gutta-percha and AH26 plus (Dentsply, DeTrey, Konstanz, Germany) using a continuous wave technique (Fig. 1C).

At the next appointment, the patient showed no significant symptoms, and therefore, porcelain fused to metal crown restoration was performed.

Eighteen months of postoperative clinical and radiographic examinations revealed no clinical signs or symptoms and no periapical pathology (Fig. 1E).

\section{Case 2}

A 30-year-old male patient was referred to the Depart- 
A

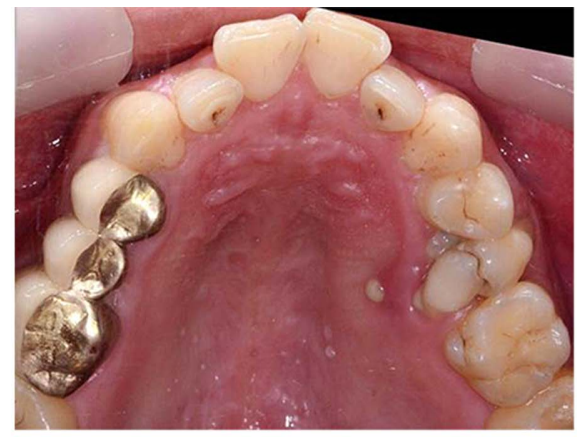

C

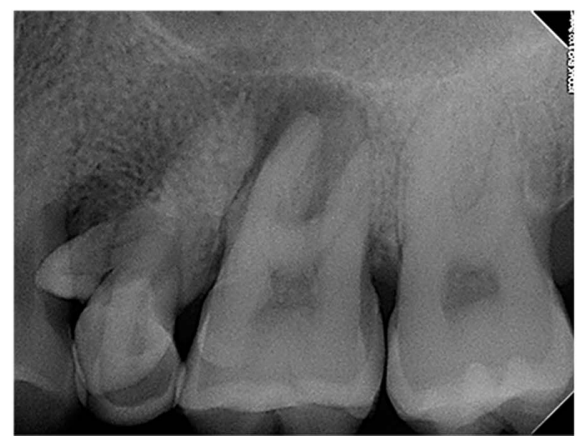

B

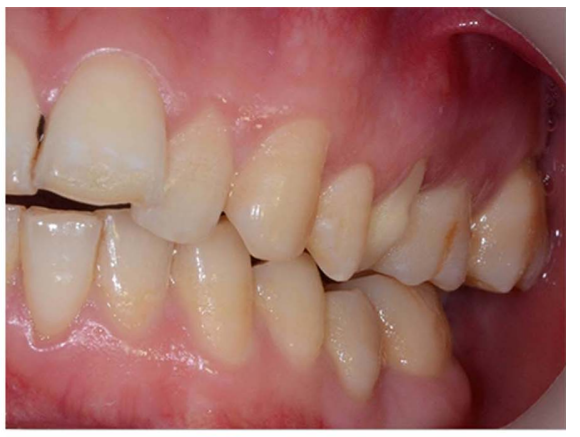

D

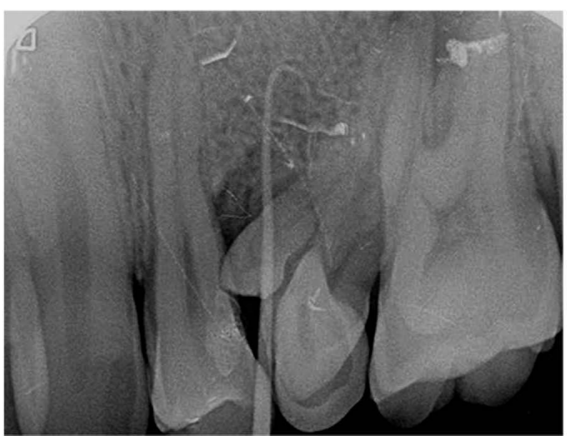

Fig. 3. (A) Preoperative clinical photograph (palatal sinus tract). (B) Preoperative clinical photograph (buccal sinus tract). (C) Preoperative periapical radiograph of the maxillary left premolar (\#25) and supernumerary teeth. (D) Gutta-percha tracing toward \#25 periapical lesion.

ment of Conservative Dentistry at Chonnam National University Dental Hospital for treatment of the left maxillary premolar (Tooth \#25) and first molar (Tooth \#26). The patient's chief complaint was gingival swelling and foul odor in the \#25 and \#26 area. The patient has a history of mental retardation and epilepsy. Clinical examination revealed a fusion associated with \#25 and two supernumerary teeth (Fig. 3A). We observed sinus tracts and gingival swelling of \#25 buccal vestibule and palatal gingival area (Fig. 3A and 3B). Periapical radiographic examination showed Gutta-percha tracing toward \#25 periapical lesion and periapical radiolucency on \#26 mesial root apex (Fig. 3D). The probing depth was over $7 \mathrm{~mm}$ on \#25 distopalatal and \#26 mesiobuccal without any mobility of \#25 and \#26.

CBCT imaging of the tooth was performed to identify the root canal system thoroughly and determine the indication for extraction or conservative treatment. Informed consent of the patient and his parents was obtained, and a CBCT of the \#25 and \#26 area was performed (Alphard VEGA, Asahi Roentgen Ind. Co.) using a tube voltage 80 $\mathrm{kV}$ and tube current of $8 \mathrm{~mA}$. The CBCT images showed separated canals in the fused tooth associated with \#25 and two supernumerary teeth, respectively (Fig. 4D). Based on these findings, the \#25 and \#26 were diagnosed with dentoalveolar abscess and conventional root canal treatment with periodontal curettage was performed after surgical flap elevation under general anesthesia.

First, informed consent for general anesthesia was obtained from the patient and his parents. Under general anesthesia, after palatal surgical flap elevation to expose distopalatal cusp, we performed access opening on \#25 and two supernumerary teeth, respectively. The working length was estimated using electronic apex locator (Denta Port RootZX) and was confirmed using a periapical radiograph (Fig. 5A). All the canals were cleaned and shaped using ProTaper NiTi rotary instruments (Protaper Universal, Dentsply) with a crown-down technique. Copious irrigation was performed using $5.25 \% \mathrm{NaOCl}$ solution and sterile saline. After canal shaping and cleaning of the fused teeth, the canals were dried and calcium hydroxide was applied as a medicament. The access cavity was temporarily sealed with Caviton (GC). We opened the access and estimated the working length followed by canal shaping and cleaning of \#26 as mentioned above (Fig. 5B). After temporary sealing of \#26 access cavity, 4-0 Mersilk suture (Ethicon, New Jersy, USA) was used for suturing the palatal flap.

At the second appointment, under general anesthesia, sinus tracts disappeared, and the canals in \#26 were filled with gutta-percha and AH26 plus (Dentsply) using a con- 
A

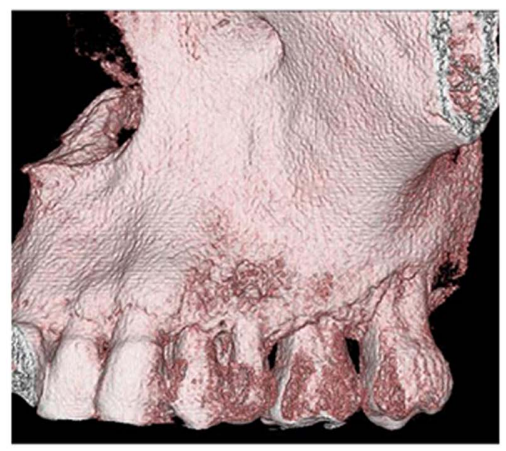

C

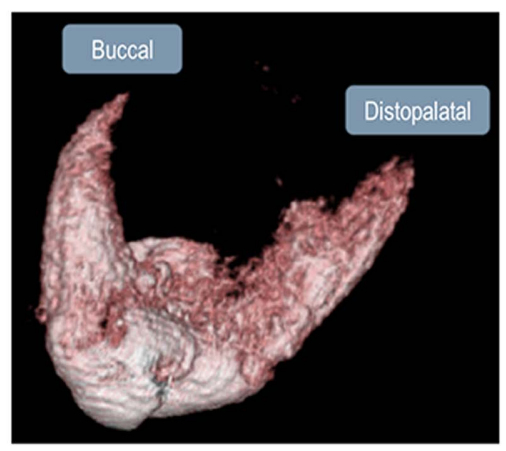

$\mathrm{E}$

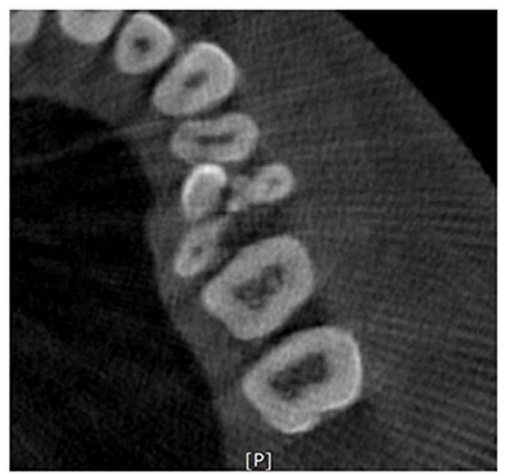

B

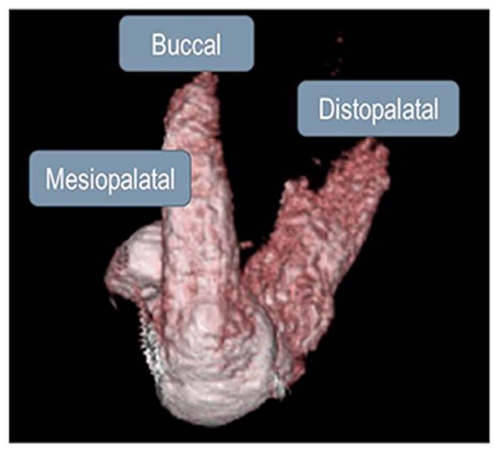

D

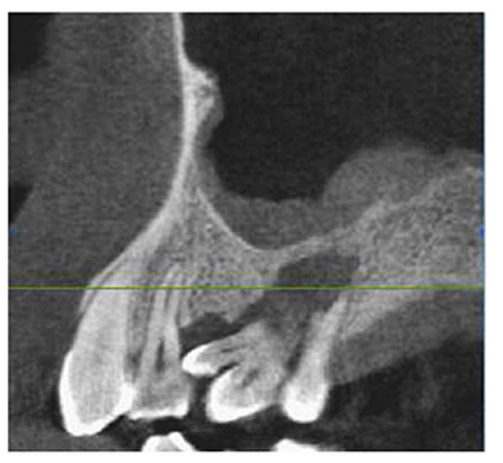

$\mathrm{F}$

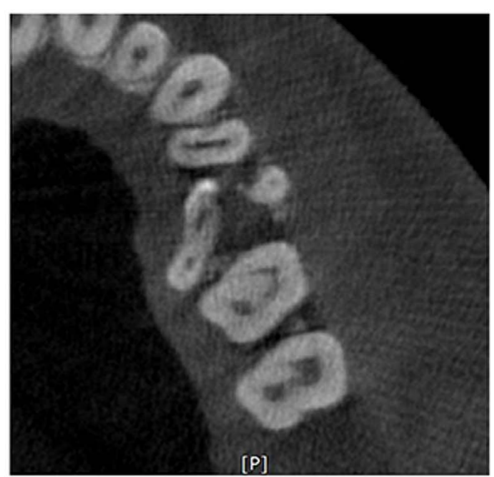

Fig. 4. CBCT images of $\# 25$ confirming the fused tooth with two supernumerary teeth. (A-C) 3-dimensional reconstruction images. (D) Sagittal image reveals separated canal of \#25 and supernumerary teeth. (E) Axial image of the coronal third. (F) Axial image of the middle third.

tinuous wave technique. Subsequently, we prepared for crown restoration of $\# 26$. We performed canal irrigation of fused tooth \#25 using $5.25 \% \mathrm{NaOCl}$, and sterile saline using an ultrasonic irrigation tip (Irrisafe, Acteon Satelec). The access cavity was sealed temporarily with caviton (GC).

At the third appointment, under general anesthesia, we found no significant clinical signs or symptoms in the patient. Therefore, we performed canal filling of the tooth as mentioned above. The access cavity of the fused tooth (\#25) was restored with LuxaCore Z (shade A3) (DMG, Hamburg, Germany). Porcelain fused to metal crown was cemented on \#26 using resin-modified glass ionomer (RelyX ${ }^{\mathrm{TM}}$ Luting Plus cement, 3M, St. Paul, MN, USA). After palatal flap elevation, root planing of the \#25 fused tooth and \#26 was performed. Palatal flap was sutured apically and repositioned using 4-0 Mersilk (Ethicon).

Clinical and radiographic examinations at 44 months postoperatively revealed no significant clinical or dental signs and symptoms or periapical pathology (Fig. 5D). However, food deposits were found on \#25 due to the morphology of crown. Therefore, we emphasized the importance of oral hygiene using appropriate tooth brushing and flossing practices.

\section{Discussion}

Usually, most mandibular first molars contain 1 mesial 
A

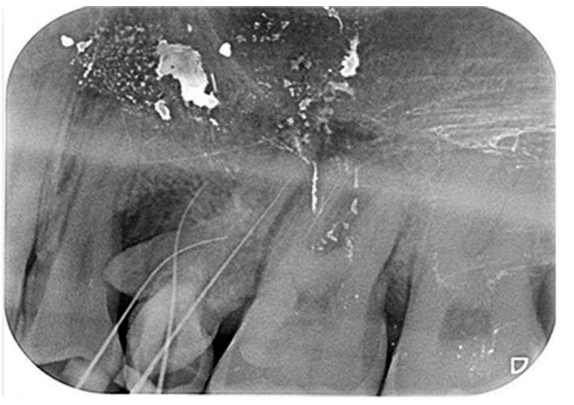

C

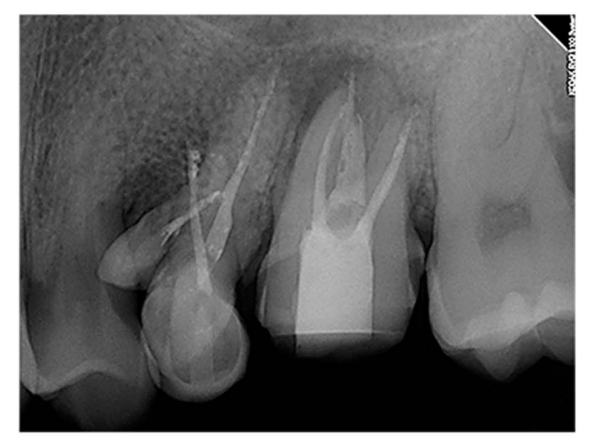

E

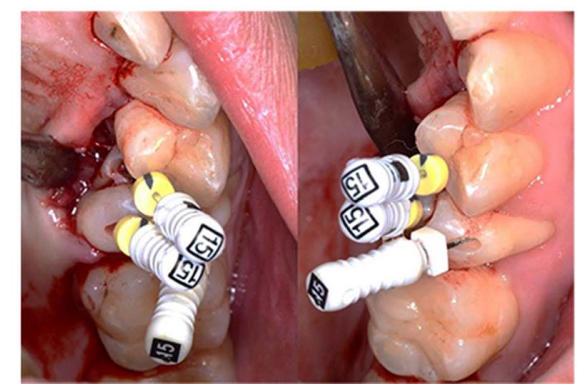

B

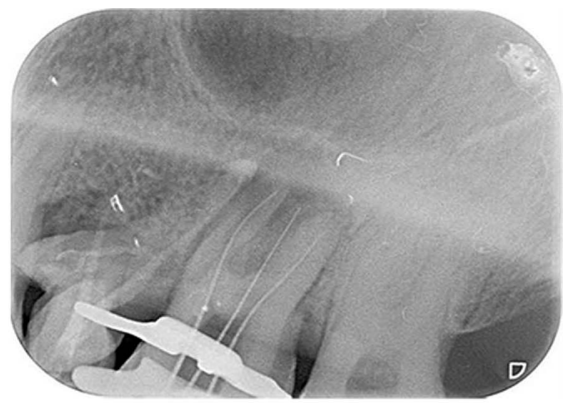

D

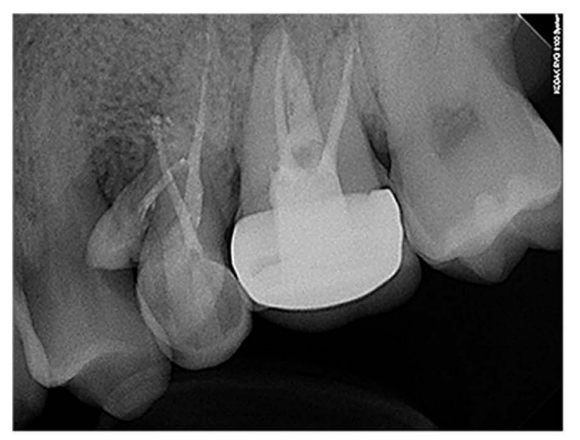

F

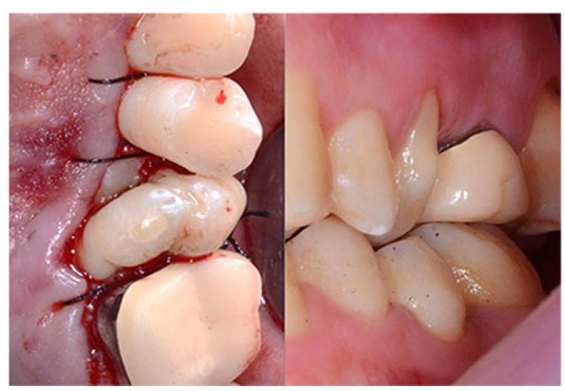

Fig. 5. (A) Periapical radiograph showing working length determination of $\# 25$ and two supernumerary teeth. (B) Periapical radiograph illustrating the working length determination of \#26. (C) Postoperative periapical radiograph. (D) 44-month follow-up periapical radiograph. (E) Clinical image illustrating working length determination of \#25 and two supernumerary teeth with open flap. (F) Postoperative clinical photograph.

root with 2 canals and 1 distal root with a single canal. An additional distolingual root of mandibular first molars has been reported, which is generally more curved and smaller than the distobuccal root [22]. The distolingual root is typically described as a conical and small root with the apex swinging toward the buccal side. Endodontic management of this root variation may present clinical challenges [23]. Based on the curvature of RE in the bucco-lingual orientation, De Moor et al. [24] classified RE into three types. Type I refers to a straight root/root canal. Type II is characterized by a root canal with initial curvature that continues as a straight root. Type III RE is defined by a root canal with a curvature in the coronal third followed by a second curve from the middle third to the apical third [24]. The RE of our first case represents a Type III curve based on De Moor's classification.
Cooke and Cox [25] described the C-shaped configuration in mandibular molars for the first time, which is most frequently encountered in the mandibular second molars. Few studies have evaluated the C-shaped canal of mandibular first molar with RE using CBCT.

Melton et al. [26] in 1991 divided the C-shaped canal into three types. However, there was no clear distinction between the categories in Melton's classification. In addition, Melton's and Manning's classifications describe only the morphology of the canal orifices, but fail to describe the variation of the $\mathrm{C}$-shaped configuration along the root length. Therefore, other classification was proposed by Fan et al. [27,28]. In our Case 1, \#46 has 3 roots with small distolingual root, and a C-shaped configuration of the mesiobuccal root canal. This case is classified as C2 (semicolon) according to Melton's classification, and Type 
3, according to Fan's classification.

Fusion of permanent and supernumerary teeth usually occurs in the anterior maxillary region, with a reported prevalence of $0.1 \%$ [29]. Fusion of normal and supernumerary teeth leads to an anomalous broad crown, and the pulp chambers and root canals might be joined or separated, depending on the stage of development at the time of union [30]. Endodontic treatment is a challenge, owing to the complex anatomy, tooth positioning, and difficulty in rubber dam isolation. In this rare case report, we describe the treatment of two supernumerary teeth fused with premolar using CBCT. Three teeth were fused including buccal-distopalatal teeth fused in the coronal $1 / 3$ (Fig. 4E) and mesiopalatal-distopalatal teeth in the middle $1 / 3$ of the root (Fig. 4F). Mesiopalatal supernumerary tooth was impacted horizontally (Fig. 4B-D).

Radiographic examination is an essential component of endodontic management. However, conventional intraoral periapical views generate only a two-dimensional image of a three-dismensional object, resulting in the superimposition of structures. Thus, endodontic applications of computed tomography (CT) were first reported by Tachibana and Matsumoto in 1990 [31]. However, conventional medical CT uses a fan-shaped beam and produces detailed images in the axial plane. However, the high dose and long scanning time of these systems render them impractical for most dental applications. Micro-CT is a highly accurate and non-destructive method for the evaluation of root canal fillings and their constituents, although it remains a research tool and is not applicable to clinical imaging in vivo [32]. On the other hand, CBCT is an effective tool for identification of root canal systems and tooth morphology in a 3D format and a higher resolution [18]. Therefore, CBCT represents a new diagnostic tool for endodontic applications. Diagnosis based on accurate analysis of root canal systems leads to positive prognosis using conventional treatments without excessive invasive approaches. Since anomalous tooth morphologies interfere with normal tooth and gingival junctions, they increase the risk of periodontitis and dental caries [33]. Therefore, the combination of root canal and periodontal treatment appears to have a positive impact on the long-term dental prognosis.

A study comparing the efficacy of the ProTaper Ni-Ti rotary file and traditional hand instrumentation of K-files and Gates-Glidden on the $\mathrm{C}$-shaped canals, revealed greater dentin removal compared with the hand instrumentation group leaving residual untreated root canal in the
ProTaper group. In the case of the ProTaper group, the time required for canal shaping was shorter than in hand instrumentation, however, the number of clinical errors was small [34]. In other words, the NiTi rotary system reduced the time required for the formation of the curved root canals while minimizing the number of random errors, and the root canal was formed more thoroughly using a hand instrument. Therefore, it is better to use a combination of the two systems. It is also important to note that the continued use of chemical agents is an important consideration because of the presence of nascent residual root surfaces. Passive ultrasonic irrigation (PUI) appears to be an adjunctive treatment for cleaning the root canal system and that PUI is more effective than syringe irrigation [35]. In our cases, non-surgical endodontic treatment based on CBCT diagnosis, ProTaper rotary system, hand instrumentation, PUI technique and periodontal treatment resulted in successful prognosis.

We used D-mode, which has the smallest field of view (FOV) of the CBCT. However, Patel et al. [36] reported that the effective dose of a small FOV is $2 \sim 3$ times higher than the FOV of a periapical film. The use of CBCT as a diagnostic tool facilitates root canal preparation at relatively low radiation dosage. However, the risks and benefits of radiation should be considered in dental practice.

\section{Conclusions}

Endodontic treatment of morphologically abnormal teeth is a challenge. However, CBCT can be used to determine the root shape and the root canal system, which enables a positive prognosis of the anomalous teeth using only non-surgical treatment without additional invasive procedures. Therefore, CBCT analysis can be considered as an effective diagnostic tool for the treatment of teeth containing complex root canals.

\section{Acknowledgements}

This study was supported by a grant (CRI 18029-1) Chonnam National University Hospital Biomedical Research Institute and the National Research Foundation of Korea (NRF) grant funded by the Korea government (MSIP) (No. 2016R1C1B1012703). 


\section{Conflict of Interest}

The authors declare that they have no competing interests.

\section{ORCID}

$\begin{array}{ll}\text { Sung-Hyeon Choi } & 0000-0002-4324-6567 \\ \text { Na-Kyung Yoon } & 0000-0003-2649-2157 \\ \text { Ji-Hyun Jang } & 0000-0002-4672-3381 \\ \text { Young-Hoon Kim } & 0000-0003-3849-8994 \\ \text { Hoon-Sang Chang } & 0000-0002-3019-1528 \\ \text { Yun-Chan Hwang } & 0000-0002-7891-9565 \\ \text { In-Nam Hwang } & 0000-0002-5388-1919 \\ \text { Won-Mann Oh } & 0000-0001-6480-6191 \\ \text { Bin-Na Lee } & 0000-0001-8017-1835\end{array}$

\section{References}

1. Slowey RR. Radiographic aids in the detection of extra root canals. Oral Surg Oral Med Oral Pathol 1974;37: 762-772. doi: 10.1016/0030-4220(74)90142-X.

2. Song M, Kim HC, Lee W, Kim E. Analysis of the cause of failure in nonsurgical endodontic treatment by microscopic inspection during endodontic microsurgery. J Endod 2011;37:1516-1519. doi: 10.1016/j.joen.2011.06. 032.

3. Calberson FL, De Moor RJ, Deroose CA. The radix entomolaris and paramolaris: clinical approach in endodontics. J Endod 2007;33:58-63. doi: 10.1016/j.joen. 2006.05.007.

4. Sperber GH, Moreau JL. Study of the number of roots and canals in Senegalese first permanent mandibular molars. Int Endod J 1998;31:117-122. doi: 10.1046/ j.1365-2591.1998.00126.x.

5. Tratman EK. Three-rooted lower molars in man and their racial distribution. Br Dent J 1938;64:74.

6. Yew SC, Chan K. A retrospective study of endodontically treated mandibular first molars in a Chinese population. J Endod 1993;19:471-473. doi: 10.1016/S00992399(06)80536-4.

7. Reichart PA, Metah D. Three-rooted permanent mandibular first molars in the Thai. Dent Oral Epidemiol 1981;9:191-192. doi: 10.1111/j.1600-0528.1981.tb01053.x.

8. Walker RT, Quackenbush LE. Three-rooted lower first permanent molars in Hong Kong Chinese. Br Dent J 1985; 159:298-299.

9. Seo MS, Park DS. C?shaped root canals of mandibular second molars in a Korean population: clinical observation and in vitro analysis. Int Endod J 2004;37:139-144. doi: 10.1111/j.0143-2885.2004.00772.x.

10. Hannah R, Kandaswamy D, Jayaprakash N. Endodontic management of a mandibular second molar with radix entomolaris: a case report. Restor Dent Endod 2014;39:132136. doi: 10.5395/rde.2014.39.2.132.

11. Miri SS, Ghorbani H, Rashed Mohassel A. Endodontic treatment of fused teeth with talon cusp. Case Rep Dent 2014;2014:738185. doi: 10.1155/2014/738185.

12. Ghoddusi J, Zarei M, Jafarzadeh H. Endodontic treatment of a supernumerary tooth fused to a mandibular second molar: a case report. J Oral Sci 2006;48:39-41. doi: 10.2334/josnusd.48.39.

13. Kaffe I, Littner MM, Begleiter A, Buchner A. Fusion of permanent molars. Quintessence Int Dent Dig 1982;13: 1237-1239.

14. Peyrano A, Zmener O. Endodontic management of mandibular lateral incisor fused with supernumerary tooth. Endod Dent Traumatol 1995;11:196-198. doi: 10.1111/ j.1600-9657.1995.tb00487.x.

15. Friedman S, Mor H, Stabholz A. Endodontic therapy of a fused permanent maxillary lateral incisor. J Endod 1984; 10:449-451. doi: 10.1016/S0099-2399(84)80267-8.

16. Mader CL. Fusion of teeth. J Am Dent Assoc 1979; 98:62-64.

17. Goldberg JM, Gross M, Rankow H. Endodontic therapy involving fused mandibular second and third molars. J Endod 1985;11:346-347. doi: 10.1016/S0099-2399(85) 80042-X.

18. Nair MK, Nair UP. Digital and advanced imaging in endodontics: a review. J Endod 2007;33:1-6. doi: 10.1016/ j.joen.2006.08.013.

19. Patel S, Kanagasingam S, Mannocci F. Cone beam computed tomography (CBCT) in endodontics. Dent Update 2010;37:373-379. doi: 10.12968/denu.2010.37.6.373.

20. La SH, Jung DH, Kim EC, Min KS. Identification of independent middle mesial canal in mandibular first molar using cone-beam computed tomography imaging. J Endod 2010;36:542-545. doi: 10.1016/j.joen.2009.11. 008.

21. Fanning B. CBCT the justification process, audit and review of the recent literature. J Ir Dent Assoc 2011; 57:256-261.

22. Vertucci FJ. Root canal anatomy of the human permanent teeth. Oral Surg Oral Med Oral Pathol 1984;58:589599. doi: 10.1016/0030-4220(84)90085-9.

23. Jerome CE, Hanlon Jr RJ. Dental anatomical anomalies in Asians and Pacific Islanders. J Calif Dent Assoc 2007; 35:631-636.

24. De Moor RJ, Deroose CA, Calberson FL. The radix entomolaris in mandibular first molars: an endodontic challenge. Int Endod J 2004;37:789-799. doi: 10.1111/ 
j.1365-2591.2004.00870.x.

25. Cooke HG, Cox FL. C-shaped canal configurations in mandibular molars. J Am Dent Assoc 1979;99:836-839. doi: 10.14219/jada.archive.1979.0402.

26. Melton DC, Krell KV, Fuller MW. Anatomical and histological features of C-shaped canals in mandibular second molars. J Endod 1991;17:384-388. doi: 10.1016/ S0099-2399(06)81990-4.

27. Fan B, Cheung GS, Fan M, Gutmann JL, Bian Z. C-shaped canal system in mandibular second molars: part I-anatomical features. J Endod 2004;30:899-903. doi: 10.1097/01. don.0000136207.12204.e4.

28. Fan B, Cheung GS, Fan M, Gutmann JL, Fan W. Cshaped canal system in mandibular second molars: Part II-Radiographic features. J Endod 2004;30:904-908. doi: 10.1097/01.don.0000136206.73115.93.

29. Velasco LF, Araujo FB, Ferreira ES, Velasco LE. Esthetic and functional treatment of a fused permanent tooth: a case report. Quintessence Int 1997;28:677-680.

30. Brook AH, Winter GB. Double teeth. A retrospective study of 'geminated' and 'fused' teeth in children. $\mathrm{Br}$ Dent J 1970;129:123-130.

31. Tachibana H, Matsumoto K. Applicability of x-ray computerized tomography in endodontics. Endod Dent Trau- matol 1990;6:16-20. doi: 10.1111/j.1600-9657.1990. tb00381.x.

32. Cotton TP, Geisler TM, Holden DT, Schwartz SA, Schindler WG. Endodontic applications of cone-beam volumetric tomography. J Endod 2007;33:1121-1132.

33. de Siqueira VC, Braga TL, Martins MA, Raitz R, Martins MD. Dental fusion and dens evaginatus in the permanent dentition: literature review and clinical case report with conservative treatment. J Dent Child 2004;71:6972.

34. Yin X, Cheung GS, Zhang C, Masuda YM, Kimura Y, Matsumoto K. Micro-computed tomographic comparison of nickel-titanium rotary versus traditional instruments in C-shaped root canal system. J Endod 2010; 36:708-712. doi: 10.1016/j.joen.2010.01.003.

35. Van der Sluis LW, Versluis M, Wu MK, Wesselink PR. Passive ultrasonic irrigation of the root canal: a review of the literature. Int Endod J 2007;40:415-426. doi: 10.1111/j.1365-2591.2007.01243.x.

36. Patel S, Dawood A, Ford TP, Whaites E. The potential applications of cone beam computed tomography in the management of endodontic problems. Int Endod J 2007; 40:818-830. doi: 10.1111/j.1365-2591.2007.01299.x. 